\title{
Chronic lymphocytic leukemia (CLL): upfront treatment options in 2017
}

\begin{abstract}
Chronic lymphocytic leukemia (CLL) is the most common adult leukemia diagnosed in the Western world. Within the past decade, significant improvement in molecular analysis leads to better understanding of the CLL biology. The heterogeneity in mutational status permits to stratify patients into different risk group categories. When treatment is indicated, the cornerstone is intensive chemoimmunotherapy. Fludarabine, cyclophosphamide and rituximab (FCR) is the standard first-line option. But its toxicity precludes its use in older patients with coexisting conditions. The combination of chlorambucil and new monoclonal anti-CD20 antibodies (obinutuzumab, ofatumumab) is an alternative option. Also patients with deletion 17p (del17p) has poor response to chemoimmunotherapy. Single agent ibrutinib is the best choice.
\end{abstract}

Volume 4 Issue 2 - 2017

\author{
Riwa Sakr, Marcel Massoud \\ Department of hematology-oncology, Holy Spirit University, \\ Lebanon
}

Correspondence: Marcel Massoud, Department of hematology-oncology, CHU-Notre Dame de Secours, Byblos, Lebanon and Faculty of medical sciences, Holy Spirit University, USEK, Kaslik, Lebanon, Tel 96I 3364359

Email marcel.massoud@gmail.com

Received: January 27, 2016 | Published: February 17, 2017

Keywords: obinutuzumab, ofatumumab, chlorambucil, idelalisib, CLL upfront treatment, ibrutinib, complement-I, NOTCH-1, CLL 11, RESONATE-2

Abbreviations: CLL, chronic lymphocytic leukemia; FCR, fludarabine, cyclophosphamide and rituximab; PI3K, phosphatidylinositol 3-kinase; IWCLL, international workshop on chronic lymphocytic leukemia; CIRS, cumulative illness rating scale; CIT, chemoimmunotherapy; FISH, fluorescence in situ hybridization; PFS, progression free survival; EMA, european medicines agency; $\mathrm{AE}$, adverse events; ORR, overall response rate; CR, complete response; $\operatorname{Ig} V H$, immunoglobulin variable heavy chain gene; ZAP, zeta-associated protein

\section{Introduction}

Chronic lymphocytic leukemia (CLL) represents the most common leukemia diagnosed in the Western world nowadays. The median age at diagnosis is 70years-old. The improved characterization of the disease through the advances in molecular tests and the better understanding of the biology of the disease have led to better risk stratification and thus to personalized treatment strategies. In the last years, several novel therapies are introduced and approved in the upfront treatment as well as in the relapse/refractory disease. These include novel CD20 monoclonal antibodies (ofatumumab and obinutuzumab), Phosphatidylinositol 3-kinase (PI3K) inhibitors (idelalisib) and Bruton tyrosine kinase inhibitors (ibrutinib). Here we reviewed the diagnosis and the risk stratification and accordingly, the available upfront treatment strategies.

\section{Diagnosis and risk stratification}

CLL is a monoclonal proliferation of mature B lymphocytes which will accumulate in bone marrow, peripheral blood and lymph nodes. ${ }^{1}$ The diagnosis is established by the presence of more than 5000 clonal B lymphocytes $/ \mathrm{mm}^{3}$ persisting for 3 months or more in peripheral blood. At flow cytometry, the corresponding phenotype is that of mature, activated B lymphocytes with co-expression of CD19, CD20, CD23 and CD5 (T cell antigen) and reduced level of membrane immunoglobulin and CD79b. Bone marrow aspirate and biopsy is not routinely done. It is helpful if unexplained cytopenia exist. Initially, lymph node biopsy is not warranted. It is indicated when transformation is suspected (Richter Syndrome). ${ }^{1}$ At diagnosis, the majority of patients are asymptomatic. Only $10 \%$ of patients present disease-related symptoms. The median age at diagnosis is around 70years-old.

While the morphological and immunological phenotypes of CLL patients are homogenous, the clinical outcome is quiet variant. Additional molecular and mutational analyses are needed to stratify patients into risk group categories. CD38 and ZAP (zeta-associated protein)-70 over expression are correlated with the unmutated $\operatorname{Ig} V H$ (immunoglobulin variable heavy chain gene) and are associated with poor prognosis. Whereas, the mutated $\operatorname{Ig} V H$ status is associated with a favorable outcome. Other recurrent genetic lesions bearing unfavorable outcome are deletions of $17 \mathrm{p}$ and $11 \mathrm{q}$ and NOTCH-1 mutation. Del1 $7 \mathrm{p}$ is found in less than ten percent of cases at diagnosis, more frequently in the unmutated $\operatorname{Ig} V H$ group. It is associated with genomic instability via the inactivation of P53. Patients are often chemo-resistant with poor prognosis., ${ }^{2,3}$ Del11q is found in $20 \%$ of cases at diagnosis. It is associated with un mutated Ig $V H$. It will inactivate the ATM (Ataxia telangiectasia mutated) gene, leading to genomic instability, chemoresistance and poor outcome. ${ }^{4} \mathrm{NOTCH}-1$ constitutes $10 \%$ of cases at diagnosis. It is associated with reduced expression of CD20 and thus reduced benefit from $\mathrm{CD} 20$ monoclonal antibody. ${ }^{5,6}$

\section{Indications for upfront treatment}

There are no potential benefits in term of survival for upfront treatment in patients with asymptomatic isolated elevated leukocyte count (Binet stage A) except when disease progression occurs. ${ }^{1}$ Whereas, intermediate and high risk patients (Binet stage B \& C) should be treated, with rare exceptions when monitoring can be an option, until evidence of disease progression (some Binet stage B). ${ }^{1}$ Symptomatic disease defined as per the 2008 International Workshop on Chronic Lymphocytic Leukemia (IWCLL), should be promptly treated: ${ }^{1}$

i. Evidence of bone marrow failure causing cytopenia

ii. Progressive splenomegaly or lymphadenopathy or bulky lymph nodes $(>10 \mathrm{~cm}$ in longest diameter) 
iii. Progressive lymphocytosis defined by lymphocyte doubling time less than 6 months or more than $50 \%$ increase in 2 -months

iv. Autoimmune anemia and/or thrombocytopenia resistant to corticosteroids or other standard therapy

v. Significant disease-related symptoms affecting quality of life (fatigue, unintentional weight loss greater than $10 \%$ within 6 months, unexplained night sweats for more than 1 month and unexplained fevers $\left(>38.0^{\circ} \mathrm{C}\right)$ for 2 weeks without evidence of infection).

\section{Upfront treatment}

When IWCLL criteria are met, first-line treatment is considered. Many factors should be measured including age, comorbidities and performance status. A co-morbidity index (cumulative illness rating scale (CIRS)) developed by the German CLL Study Group would categorize patients to be fit for intensive chemoimmunotherapy (CIT) when the CIRS $\leq 6$ and creatinine clearance $\geq 70 \mathrm{~mL} / \mathrm{min}$ or to be frail and unfit for intensive CIT. ${ }^{7}$ Other factors affecting the choice of treatment are unfavorable predictive biomarkers. Del17p and del11q by fluorescence in situ hybridization (FISH) are chemo-resistant and would benefit from no chemotherapy approaches. Other high risk feature is NOTCH1 mutation which has reduced benefit from rituximab. In this review, we will categorize patients according to the CIRS and the presence or not of unfavorable molecular prognostic factors to three groups: Patients with del17p, frail patients without del17p and fit patients without del17p.
Patients with del17p: CLL patients with del17p/TP53 mutation have an aggressive disease behavior. It responds poorly to chemoimmunotherapy. The median progression free survival (PFS) is 11.3months when treated with FCR and 7.9 months with BendamustineRituximab (BR). ${ }^{8,9}$ A humanized anti-CD52 monoclonal antibody $(\mathrm{mAb})$, Alemtuzumab was compared to chlorambucil in the upfront treatment of CLL patients with del17p. The median PFS was 10.7 months similar to the median PFS achieved with FCR. It was associated with serious infections such as Cytomegalovirus reactivation due to T-cell depletion. Consequently, it was withdrawn from the market. ${ }^{10}$ Ibrutinib, an oral selective and irreversible BTK inhibitor is one of the novel agents extensively studied and approved in relapse/refractory CLL patients as well as in del17p. In a phase II trial, Farooqui et al. ${ }^{11}$ reported in 2015 the results of 35 treatmentnaïve patients' with CLL del17p treated with single agent ibrutinib. ${ }^{11}$ The ORR was $97 \%$, including $12 \%$ CR and $70 \%$ PR. PFS was $91 \%$ at 2-years. Based on these impressive results, the drug was approved in the first-line setting of CLL patients with del17p. Idelalisib, a phosphatidylinositol-3 kinase inhibitor, in association with rituximab, is approved in patients with relapse/refractory CLL. O'Brian et al. ${ }^{12}$ reported in 2014 the results of 64 treatment-naïve older CLL patients, of which 9 patients had del17p/TP53 mutation. The ORR was $97 \%$ at the expense of important toxicities, including late-onset grade 3 diarrhea/colitis in $42 \%$ of cases. For the moment, this combination is only approved by the European Medicines agency (EMA) for del17p in the first-line setting (Table 1). ${ }^{12}$

Table I Summary of upfront therapy for CLL patients with deletion I7p or TP53 mutation

\begin{tabular}{|c|c|c|c|c|c|c|c|}
\hline Agent & Study & & $\begin{array}{l}\text { Number of } \\
\text { patients }\end{array}$ & $\begin{array}{l}\text { Number of patients with } \\
\text { Del(I 7p) /TP } 53 \text { mutation }\end{array}$ & ORR & CR & $\begin{array}{l}\text { PFS at } \\
2 y\end{array}$ \\
\hline Ibrutinib & Farooqui et al."' & Phase II & 51 & 35 & $97 \%$ & $12 \%$ & $91 \%$ \\
\hline Idelalisib + Rituximab & O'brien et al. ${ }^{12}$ & Phase II & 64 & 9 & $97 \%$ & $33 \%$ & $92 \%$ \\
\hline
\end{tabular}

Frail patients without del17p: Historically, the standard treatment for this group of patient was chlorambucil. In the era of new monoclonal antibodies, two recent studies 'CLL 11' and 'complement 1 ' have evaluated the outcome of adding, to chlorambucil, one of the available anti CD20 antibodies (rituximab, obinutuzumab and ofatumumab). ${ }^{13,14}$ Moreover, RESONATE-2 study, in the absence of del17p, compared ibrutinib to chlorambucil in untreated CLL patients aged $>65$ years with comorbidities. ${ }^{15} \mathrm{~A}$ randomized phase III study (CLL 11-trial) conducted by the GCLLSG, compared obinutuzumab (a humanized type II CD20 mAb) plus chlorambucil to rituximab (type I CD20 mAb) plus chlorambucil to the current standard firstline treatment chlorambucil monotherapy. ${ }^{13} 781$ previously untreated CLL patients with CIRS score $>6$ and/or creatinine clearance $<70 \mathrm{ml} /$ min were enrolled. The median age was 73years. Less than $10 \%$ of patients had Del 17p. ORR was higher in obinutuzumab-chlorambucil arm compared to rituximab-chlorambucil and to chlorambucil alone ( $78 \%$ vs. $65 \%$ vs. $31 \%$, p $<0.001$ ). CR was achieved in $20.7 \%$ vs. $7.0 \%$ vs. $0 \%$ respectively and median PFS was 26.7 vs. 16.3 vs. 11.1 months $(p<0.001)$ respectively. There were no statistically PFS advantages for the subgroup of patient with Del $17 \mathrm{p}$. The only OS advantage was observed when obinutuzumab-chlorambucil was compared to chlorambucil monotherapy. Obinutuzumab-chlorambucil arm had an acceptable safety profile, with more infusion related adverse events (AE) occurring in the first cycle $(20 \%$ vs $4 \%$ in the rituximabchlorambucil). Neutropenia was most prevalent in the obinutuzumabchlorambucil (33\%) compared to rituximab-chlorambucil (28\%).
In patients ineligible for intensive CIT, another phase III trial (Complement-1 trial), evaluated 447 naïve patients with active CLL to receive chlorambucil \pm Ofatumumab (type I CD20mAb).${ }^{14}$ The median age was 69 years. The ofatumumab-chlorambucil arm demonstrated superiority over chlorambucil monotherapy in term of ORR $(82 \%$ vs. $69 \%, p<0.001)$, CR $(14 \%$ vs. $1 \%)$ and median PFS (22.4 vs. 13.1months, $\mathrm{p}<0.001)$. There was no statistically PFS advantage for the subgroup of patients with Del 17p. No survival benefit was observed. The ofatumumab-chlorambucil arm had acceptable safety profile, with neutropenia being the most common ( $26 \%$ vs $14 \%$ in the chlorambucil arm). Mild infusion-related side effects were also noted in the ofatumumab-chlorambucil arm.

Ibrutinib is approved in relapse/refractory CLL patients as well as in del17p. In the first-line setting, Resonate- 2 trial, a randomized phase III study, evaluate the efficacy and safety of single agent ibrutinib compared to chlorambucil in 269 CLL patients aged 65 years or older with coexisting conditions. ${ }^{15}$ The median age was 73 years. Patients with del17p were excluded. Ibrutinib monotherapy demonstrated better efficacy than chlorambucil in term of ORR ( $86 \%$ vs. $35 \%$, p $<0.001)$, PFS (median PFS, NR vs. 18.9 months, $\mathrm{HR}=0.16, \mathrm{p}<0.001$ ) and $\mathrm{OS}$ (24 months-OS $98 \%$ vs. $85 \%$, HR $=0.16, \mathrm{p}=0.001$ ). Most common side effects were diarrhea, cough, fatigue and nausea in ibrutinib arm. A grade 3 hemorrhage was noted in 3 patients and grade 4 in one patient. In summary, in frail treatment-naïve patients without del17p, antiCD20 antibodies-based CIT is associated with better efficacy than 
chlorambucil monotherapy in term of PFS. Survival advantage was seen only obinutuzumab was associated with chlorambucil. To date, in patients with Del $17 \mathrm{p}$, there was no advantage when anti-CD20 antibody was added to chlorambucil. Finally, single agent ibrutinib demonstrates better efficacy than chlorambucil in upfront setting with acceptable toxicities (Table 2).

Table 2 Summary of upfront therapy for frail CLL patient without Del (17p)

\begin{tabular}{llllllll}
\hline Agent & Study & & $\begin{array}{l}\text { Number of } \\
\text { patients }\end{array}$ & $\begin{array}{l}\text { Median age } \\
(\mathbf{y r})\end{array}$ & ORR & CR & Median PFS \\
\hline G-Cbl (arm) & CLL-II trial & Phase III & 238 & 74 & $78 \%$ & $20.70 \%$ & 26.7 months \\
R-Cbl (arm) & CLL-II trial & Phase III & 233 & 73 & $65 \%$ & $7 \%$ & 16.3 months \\
O-Cbl (arm) & COMPLEMENT I & Phase III & 22 I & 69 & $82 \%$ & $14 \%$ & 22.4 months \\
Oral ibrutinib & RESONATE 2 & Phase III & 136 & 73 & $86 \%$ & $4 \%$ & NR \\
\hline
\end{tabular}

Fit patients without del17p: The current standard of care for initial therapy in this group category is CIT with Fludarabine, cyclophosphamide and rituximab (FCR protocol). ${ }^{16}$ It was initially described by the MD Anderson group and the GCLLSG. Tam et al. ${ }^{17}$ showed the results of long-term outcomes of 300 patients with upfront FCR treatment. The median age was 57years. The overall response rate (ORR) was $95 \%$ with $72 \%$ complete response (CR) compared to $51 \%$ in older patients (>70years). Progression-free survival was 80 months in patients with partial response. Twenty-five percent of patients discontinued treatment before achieving the six cycles because of adverse events, persistent cytopenia being the most prevalent. ${ }^{17}$ The German group GCLLSG in a phase III trial (CLL8-trial) compared, FCR to FC (Fludarabine, cyclophosphamide) as initial treatment in patients without previous comorbidities (CIRS $\leq 6$ Creatinine clearance $\geq 70 \mathrm{ml} / \mathrm{min}$ ). The median age was 61years. At three years' followup, overall survival was statistically improved with the addition of rituximab ( $87 \%$ in FCR group vs. $83 \%$ in $\mathrm{FC}(\mathrm{p}=0.012)$ ), median PFS was 52 months (FCR) compared to 33 months (FC) $(\mathrm{p}<0.0001)$ and $\mathrm{CR}$ was achieved in $44 \%$ in FCR group compared to $22 \%$ in FC group $(\mathrm{p}<0.0001){ }^{8}$

Bendamustine, an alkylating agent, in combination with rituximab (BR) was also studied in previously untreated CLL patients. The GCLLSG showed in a phase II trial, the outcomes of 117 treatmentnaive CLL patients, treated with combined Bendamustine $\left(90 \mathrm{mg} / \mathrm{m}^{2}\right.$ on 2 consecutive days) and rituximab $\left(375 \mathrm{mg} / \mathrm{m}^{2}\right.$ on day 0 of the first cycle, followed by $500 \mathrm{mg} / \mathrm{m}^{2}$ on subsequent courses). ${ }^{9}$ The median age was 64 years. Inclusion criteria were creatinine clearance $\geq 30 \mathrm{ml} /$ min. The ORR was $88 \%$ with $23 \% \mathrm{CR}$. Patients with creatinine clearance less than $70 \mathrm{~mL} / \mathrm{min}$ (ineligible for FCR protocol), did as good as those with more than $70 \mathrm{~mL} / \mathrm{min}$. The median PFS was 34 months. $26 \%$ of patients were older than 70 years of age and had similar PFS compared to young patients. In a randomized phase III trial comparing FCR to BR in a first-line setting of fit CLL patients, the GCLLSG showed a superiority of FCR compared to BR in term of CR (39.7 vs. 30.8, p=0.03) and PFS (median PFS 55.2 vs. 41.7 months, $p<0.001) .^{7}$ There was no difference in OS between the 2 arms. Grade 3 and 4 adverse events were higher in FCR group but treatment-related mortality was similar in both groups. On the other hand, in a subgroup analysis of patients $>65$ years of age, median PFS was comparable between FCR and BR, at the expense of increased risk of infections $(47.7 \%$ vs. $20.6 \%, p<0.001)$. Based on these results, the upfront treatment for fit CLL patients is FCR. Nevertheless, BR can be administered in older patients ( $>65$ years) as well as in patients with altered renal function (creatinine clearance $30-70 \mathrm{~mL} / \mathrm{min}$ ) where FCR can cause more cytopenia leading to reduce dosing and further treatment discontinuation.

As discussed previously, RESONATE-2 study has demonstrated the efficacy of single agent ibrutinib in patients older than 65years without Del $17 \mathrm{p}$. To resume, previously untreated CLL patients, presenting no comorbidities (CIRS $\leq 6$ and creatinine clearance $\geq 70 \mathrm{~mL} / \mathrm{min}$ ), in whom treatment indications meet the IWCLL criteria are eligible for CIT with the standard of care protocol FCR. Whereas, $\mathrm{BR}$ and ibrutinib can be an option especially for fit patients with $>65$ years of age (Table 3 ).

Table 3 Summary of upfront therapy for fit CLL patients without Del 17p

\begin{tabular}{|c|c|c|c|c|c|c|c|}
\hline Agent & Study & & $\begin{array}{l}\text { Number of } \\
\text { patients }\end{array}$ & $\begin{array}{l}\text { Median age } \\
(y r)\end{array}$ & ORR & CR & Median PFS \\
\hline \multirow{2}{*}{ FCR (arm) } & CLL- 10 trial & \multirow{2}{*}{ Phase III } & 282 & 61 & $95.40 \%$ & $44 \%$ & 55.2 months \\
\hline & Patient $>65 y r$ & & 88 & -- & -- & -- & 45.6 months \\
\hline \multirow{2}{*}{ BR (arm) } & CLL-10 trial & \multirow{2}{*}{ Phase III } & 279 & 62 & $95.70 \%$ & $30.80 \%$ & 41.7 months \\
\hline & Patient $>65 y \mathrm{r}$ & & 108 & -- & -- & -- & NR \\
\hline Oral ibrutinib & RESONATE 2 & Phase III & 136 & 73 & $86 \%$ & $4 \%$ & NR \\
\hline
\end{tabular}

\section{Conclusion}

We are witnessing a shift in the treatment algorithm in the first-line setting of CLL patients. In the era of novel therapies, we are moving from one-regimen fits all patients to more personalized approach. Selection of patients depends on age, co-morbidity and del17p. FCR is still the cornerstone for young fit patients whereas BR is more suitable for fit older patients. The combination of anti-CD20mAb with chlorambucil is the preferred option in frail older patients. Oral ibrutinib demonstrates its efficacy in del17p as well as in older patients with coexisting conditions without del17p due to acceptable toxicity profile. Idelalisib in association with rituximab has been approved by the EMA in the upfront treatment of patients with del17p (Figure 1). Several ongoing studies will clarify the role of new targeted therapies in the initial treatment of CLL. 


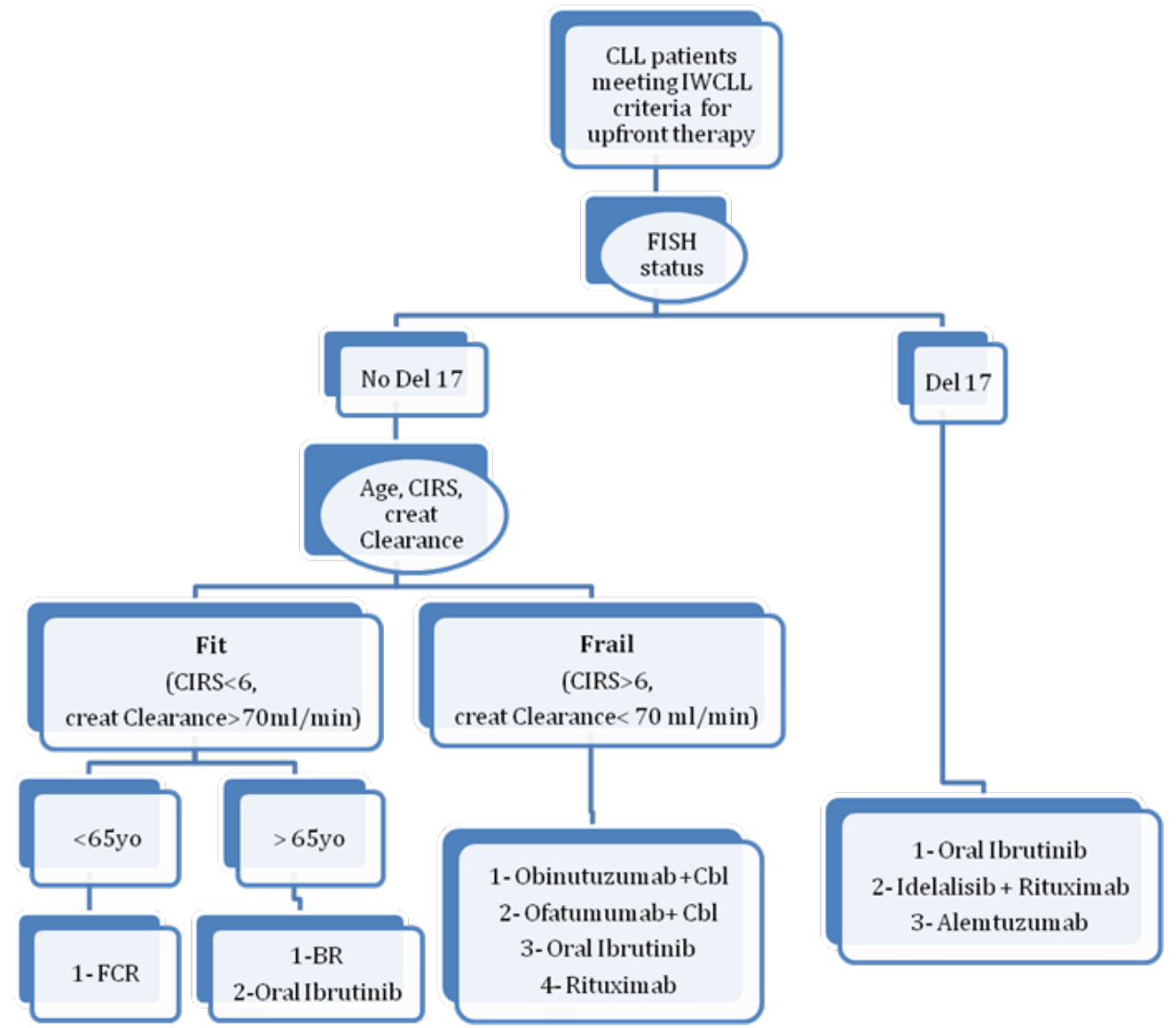

Figure I Suggested Treatment Algorithm for upfront therapy in CLL patient.

\section{Acknowledgements}

None.

\section{Conflict of interest}

The author declares no conflict of interest.

\section{References}

1. Hallek M, Cheson BD, Catovsky D, et al. Guidelines for the diagnosis and treatment of chronic lymphocytic leukemia: a report from the International workshop on chronic lymphocytic leukemia updating the national cancer institute working group 1996 guidelines. Blood. 2008;111(12):5446-5456.

2. Döhner H, Stilgenbauer S, Benner A, et al. Genomic aberrations and survival in chronic lymphocytic leukemia. $N$ Engl $\mathrm{J} \mathrm{Med}$. 2000;343(26):1910-1916.

3. Wattel E, Preudhomme C, Hecquet B, et al. p53 mutations are associated with resistance to chemotherapy and short survival in hematologic malignancies. Blood. 1994;84(9):3148-3157.

4. Stankovic T, Skowronska A. The role of ATM mutations and $11 \mathrm{q}$ deletions in disease progression in chronic lymphocytic leukemia. Leuk Lymphoma. 2014;55(6):1227-1239.

5. Pozzo F, Bittolo T, Arruga F, et al. NOTCH1 mutations associate with low CD20 level in chronic lymphocytic leukemia: evidence for a NOTCH1 mutation-driven epigenetic dysregulation. Leukemia. 2015;30(1):182189.

6. Stilgenbauer S, Schnaiter A, Paschka P, et al. Gene mutations and treatment outcome in chronic lymphocytic leukemia: results from the CLL8 trial. Blood. 2014;123(21):3247-3254.
7. Eichhorst B, Fink AM, Busch R, et al. Frontline chemoimmunotherapy with fludarabine $(\mathrm{F})$, cyclophosphamide $(\mathrm{C})$ and rituximab (R) (FCR) shows superior efficacy in comparison to bendamustine (B) and rituximab (BR) in previously untreated and physically fit patients (pts) with advanced chronic lymphocytic leukemia (CLL): final analysis of an international, randomized study of the German CLL Study Group (GCLLSG) (CLL10 Study). Blood. 2014;124(21):19.

8. Hallek M, Fischer K, Rowson GF, et al. Addition of rituximab to fludarabine and cyclophosphamide in patients with chronic lymphocytic leukaemia: a randomised, open- label, phase 3 trial. Lancet. 2010;376(9747):1164-1174.

9. Fischer K, Cramer P, Busch R, et al. Bendamustine in combination with rituximab for previously untreated patients with chronic lymphocytic leukemia: a multicenter phase II trial of the German chronic lymphocytic leukemia study group. J Clin Oncol. 2012;30(26):3209-3216.

10. Osterborg A, Foà R, Bezares RF, et al. Management guidelines for the use of alemtuzumab in chronic lymphocytic leukemia. Leukemia. 2009;23(11):1980-1988.

11. Farooqui MZ, Valdez J, Martyr S, et al. Ibrutinib for previously untreated and relapsed or refractory chronic lymphocytic leukaemia with TP53 aberrations: a phase 2, single-arm trial. Lancet Oncol. 2015;16(2):169176.

12. O’Brien S, Lamanna N, Kipps TJ, et al. Update on a Phase 2 Study of Idelalisib in Combination with Rituximab in Treatment-Naïve Patients $\geq 65$ Years with Chronic Lymphocytic Leukemia (CLL) or Small Lymphocytic Lymphoma (SLL). Blood. 2014;124(21):1994.

13. Goede V, Fischer K, Busch R, et al. Obinutuzumab plus chlorambucil in patients with CLL and coexisting conditions. $N$ Engl J Med. 2014;370(12):1101-1110. 
14. Hillmen P, Robak T, Janssens A, et al. Ofatumumab + chlorambuci versus chlorambucil alone in patients with untreated chronic lymphocytic leukemia (CLL): results of the phase III study complement 1 (OMB110911). Blood. 2013;122(21):528.

15. Burger JA, Tedeschi A, Barr PM, et al. Ibrutinib as initial therapy for patients with chronic lymphocytic leukemia. $N$ Engl J Med 2015;373(25):2425-2437.
16. Keating MJ, O'Brien S, Albitar M, et al. Early results of a chemoimmunotherapy regimen of fludarabine, cyclophosphamide and rituximab as initial therapy for chronic lymphocytic leukemia. J Clin Oncol. 2005;23(18):4079-4088.

17. Tam CS, O'Brien S, Wierda W, et al. Long-term results of the fludarabine, cyclophosphamide and rituximab regimen as initial therapy of chronic lymphocytic leukemia. Blood. 2008;112(4):975-980. 